\title{
Phosphoproteins and the Dawn of Functional Phenotyping
}

\author{
J. Bodo E.D. Hsi \\ Department of Clinical Pathology, Cleveland Clinic, Cleveland, Ohio, USA
}

\section{Key Words}

Phosphoprotein - Immunohistochemistry •

Immunofluorescence · Hematopathology • Quantification • Quantum dots

\begin{abstract}
Phosphorylation is one of the most important processes in cell signal transduction. Detection of phosphorylated proteins in cancer tissue is useful for prognosis and diagnosis, and it might be very helpful in monitoring treatment using targeted therapy. For these reasons, the in situ quantitative measurement and subcellular localization of phosphoproteins will likely be important. However, phosphoproteins are extremely labile, a likely explanation for inconsistent or contradictory reports. Thus, the development of new paradigms for tissue handling, immunostaining, and quality control are needed.

Copyright $\odot 2011$ S. Karger AG, Basel
\end{abstract}

\section{Introduction}

The modern diagnosis and associated prognosis of cancer relies in large part on tissue biopsy and analysis in the anatomic pathology laboratory. Traditional morphologic assessment with the aid of immunohistochemistry (IHC) allows diagnosis and subclassification of tumors with high accuracy. In addition, the assessment of certain biomarkers may further guide therapy. For example, assessment of breast carcinoma cells for estrogen receptor, progesterone receptor, and human epidermal receptor type 2 (Her-2) has become routine and guides therapy. In hematopathology, lack of CD20 may influence the use of rituximab in B-cell lymphoma therapy.

Molecular genetic abnormalities that help define pathologic entities as well as dictate specific therapies are increasingly being discovered. Arguably, hematologic malignancy is among the most advanced areas in this regard. The use of molecular techniques to further refine the diagnostic and prognostic groups is still evolving and will undoubtedly increase in the future. However, we believe there is an important role for pathologists to play in expanding the applications of slide-based in situ techniques. As opposed to the traditional assessment of static markers that define lineage or differentiation/maturational stages, the assessment of functional biological pathways identified by posttranslational protein modification as diagnostic, prognostic, and predictive biomarkers is in its infancy. In this article, we will focus on protein phosphorylation events and the quantitative assessment of phosphoproteins (PPs) in tissues and illustrate the types of preliminary studies and enabling technologies that must be developed to make such assays a reality in translational and diagnostic pathology.

\begin{tabular}{ll}
\hline KARGER & (c) 2011 S. Karger AG, Basel \\
Fax +41 61 306 $1234-2008 / 11 / 0782-0115 \$ 38.00 / 0$ \\
$\begin{array}{l}\text { E-Mail karger@karger.ch } \\
\text { www.karger.com }\end{array}$ & $\begin{array}{l}\text { Accessible online at: } \\
\text { www.karger.com/pat }\end{array}$
\end{tabular}




\section{Importance of PPs}

Protein phosphorylation was discovered more than 65 years ago [1]. Phosphorylation and dephosphorylation of proteins is involved in regulating nearly all cellular functions. Phosphorylation states can determine key properties of proteins including enzyme activity, protein-protein physical interactions, protein-nucleic acid physical interactions, and subcellular localization. Cell surface receptors, intracellular signaling molecules, transcription factors, cytoskeletal proteins, cellular respiratory proteins, cell cycle proteins, stress response, and apoptosisrelated proteins all have many examples of regulation by phosphorylation events, demonstrating the importance of this posttranslational modification in cell biology. By necessity, protein phosphorylation is a dynamic process determined by a complex interplay of kinases and phosphatases. This complexity is reflected by the existence of hundreds of these enzymes [2].

\section{Applications of PP Detection in Diagnosis and Prognosis Assessment}

Phosphoproteomic studies of tissues show promise for the discovery of key PPs or signaling pathways. Using such an approach, unknown PPs that had not been previously implicated in the genesis of the disease can be identified. While this is useful in research applications, a more limited analysis based on these discoveries is suitable for further investigation as diagnostic, prognostic, or predictive applications. Indeed, PP analysis in patient tissue samples has been shown to be useful in diagnosis and possibly in the prediction of the prognosis. An illustrative example of PP detection can be found in the immunohistochemical staining of a phosphorylated form of signal transducers and activators of transcription 5 (pSTAT5) in various hematologic malignancies. STAT transcription factors are phosphorylated and thus activated by the family of nonreceptor Janus tyrosine kinases (JAKs), and this pathway has been implicated in a variety of hematologic disorders as well as solid tumors [3]. Oncogenic fusion proteins of JAK2 with ETV6, PCM1, or BCR have been described in different leukemias and myeloproliferative disorders [4]. An activating mutation of JAK2 (JAK2 $\mathrm{V} 617 \mathrm{~F}$ ) is present in nearly all cases of polycythemia vera and in a substantial proportion of cases of essential thrombocythemia, idiopathic myelofibrosis, and refractory anemia with ringed sideroblasts and thrombocytosis $[5,6]$.
We hypothesized that constitutive activation of the JAK2 pathway as a consequence of this mutation might be manifested by an abnormal distribution of PSTAT5 in bone marrow biopsies from JAK2 V617F+ cases. Indeed, an abnormal nuclear megakaryocytic (nMEG) pSTAT5 expression was seen. Clinically, nMEG pSTAT5-positive patients seemed to require cytoreductive therapy more often than those without nMEG pSTAT5 expression [7]. Thus pSTAT5 IHC can be used for the identification of patients with the JAK2 V617F mutation. Such information can only be obtained by in situ methods like IHC that allow the evaluation of specific cell types in tissues as well as the subcellular localization of signal. In theory, other mutations capable of activating this pathway might yield a similar pattern. Alternate activating mutations in non-chronic myeloid leukemia (CML) myeloproliferative neoplasms have been described in JAK2 V617F-negative cases. For example, an activating MPL W515L mutation has been described that also activates the JAKSTAT pathway. When stained for pSTAT5, the same abnormal megakaryocytic pSTAT5 expression pattern was seen [8]. Thus, disease-specific PP IHC tissue patterns appear to be present that reflect common biological pathway perturbations despite different underlying genetic events. In principle, demonstrating altered final common pathways (through 'functional phenotyping') may be more useful than searching for multiple disparate genetic mutations.

Another kinase which plays an important role in STAT protein activation, including STAT5, is the cell surface receptor tyrosine kinase fms-like tyrosine kinase 3 (FLT3) [9]. Activating mutations in FLT3 are common in acute myeloid leukemia (AML) [10-12]. Importantly, patients with these mutations have been demonstrated to have an unfavorable prognosis in normal karyotype AML [13]. Demonstration of pSTAT5 in AML blasts by IHC might be a way to determine whether this signaling pathway is activated. Indeed, we observed that pSTAT5 in AML blasts with FLT3/ITD mutation as assessed by IHC in bone marrow biopsies is particularly strong compared to AML blasts with wild-type FLT3 [14]. We confirmed these qualitative data and using immunofluorescent assay showed a significantly higher nuclear expression of pSTAT5 in FLT3/ITD-positive cases compared to FLT3 wild-type cases [15]. In a similar line of thought, we studied another disease with constitutive tyrosine kinase activation, i.e. systemic mastocytosis (SM). Activating mutations of KIT, which again signals via STAT5, are present in all forms of SM. We found that all SM patients expressed abnormal nuclear PSTAT5 in bone marrow mast 
Fig. 1. Cellular signaling pathways involved in proliferation, differentiation, and angiogenesis in tumor cells with the indication of actions of currently approved small-molecule inhibitors for hematological malignancies.

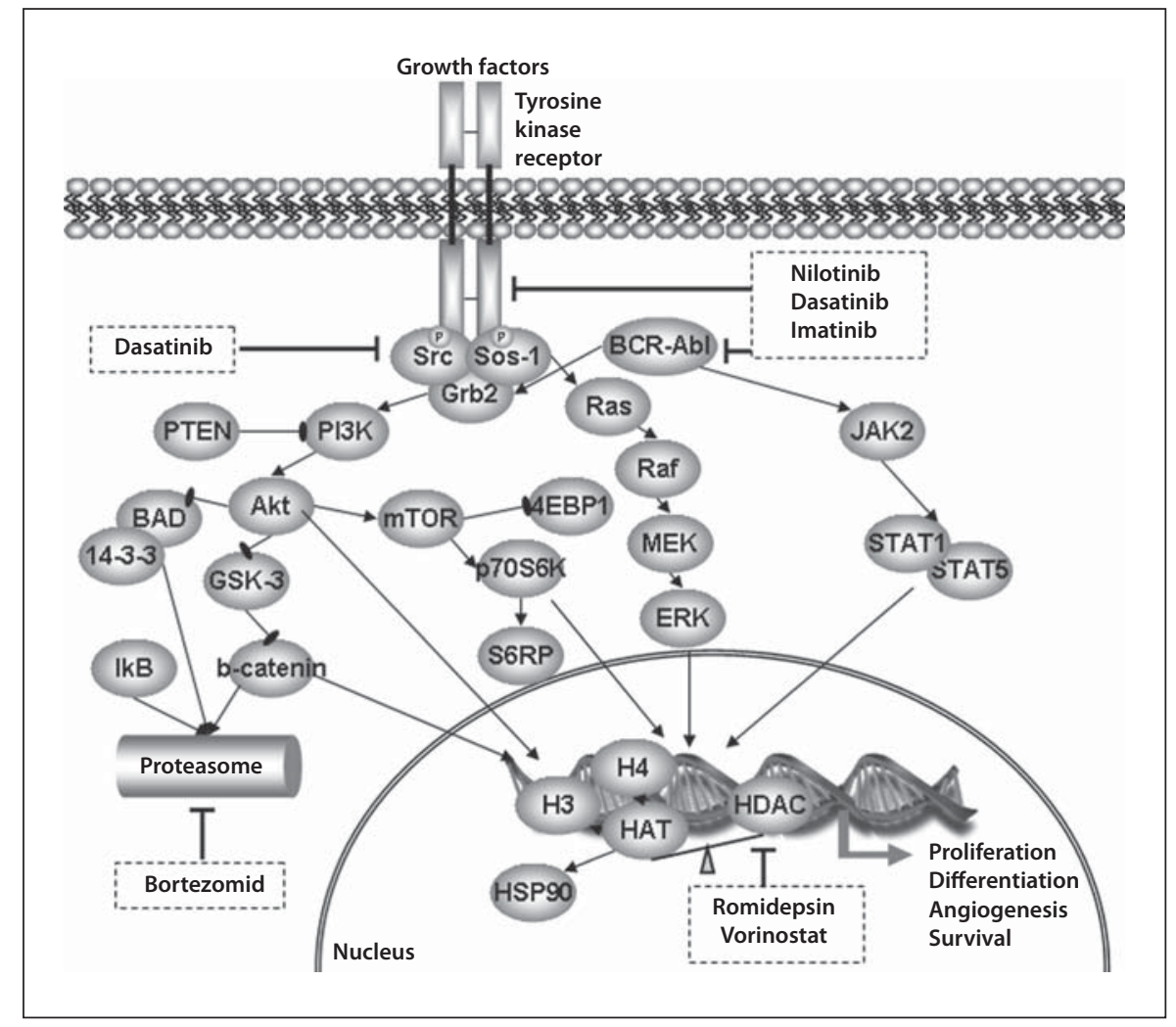

cells (MCs), while most cutaneous mastocytosis cases (that lack activating KIT mutations) and all nonneoplastic MCs lacked pSTAT5. Furthermore, abnormal pSTAT5 expression was also detected in a case of SM with eosinophilia with a FIP1L1-PDGFRA fusion. Thus, nuclear pSTAT5 in MCs appears to be a relatively robust phenotypic marker of SM [16].

\section{Monitoring of PPs in Tissue in the Context of Targeted Therapies}

Targeted therapies have significantly changed the treatment of cancer over the past decade. Monoclonal antibodies and small-molecule inhibitors are now important parts of many cancer therapies, including those for leukemia, lymphoma, and multiple myeloma as well as breast, lung, colorectal, and pancreatic cancers. Functional phenotyping for specific PPs would allow the assessment of key cellular pathways that might justify the use of a particular targeted therapy. If validated in clinical studies, tailoring therapy by identifying abnormally active signaling pathways is possible. Furthermore, moni- toring of drug effects in tumor cells might also be useful as surrogates of efficacy.

Likely candidates for PP analysis in order to monitor targeted therapy are phosphorylated (activated) kinases such as Src, ERK, AKT, or mTOR and their downstream targets (fig. 1). For example phospho-Src (pSrc) was proposed as a biomarker for dasatanib therapy [17], which is specifically indicated to treat adults with chronic, accelerated, or myeloid or lymphoid blast phase CML with resistance or intolerance to prior therapy including imatinib. Dasatinib inhibits multiple tyrosine kinases, including BCR-ABL, Src family kinases, c-KIT, EPHA2, and PDGFR $\beta$ [18-21]. Furthermore, pSrc inhibition in surrogate tissue PBMCs showed a similar relationship with inhibition of Src kinase activity. This is particularly relevant when pSrc in PBMCs is used as a biomarker for clinical studies in which the availability of tumor biopsies is usually limited. When the inhibition of pSrc was compared with tumor growth inhibition by dasatinib, pSrc levels in both tumors and PBMCs correlated well with the preclinical in vivo efficacy [17]. 


\section{Challenges in PP Assessment}

PP detection and quantification represents a new area for diagnostic pathology that exploits specific functional characteristics of cells within the context of a tissue section. Specific cells within a mixed tumor sample can be assessed for expression as well as subcellular localization, both of which have functional significance. However, PPs are extremely labile, reflecting dynamic processes. Thus, their accurate detection will require anatomic pathology laboratories to develop new paradigms for tissue handling, immunoassay development, and quality control.

In order to have a useful assay several conditions must be met. These include: (1) ensuring tissue handling procedures and fixatives maintain the in vivo PP status, (2) demonstrating that the PP antibody is specific, (3) generation of robust control tissue samples, (4) selection of an appropriate imaging system for quantitative analysis, and (5) demonstration of acceptable performance characteristics (sensitivity/specificity for a qualitative assay or $\mathrm{CV}$, limits of detection, and working range for quantitative assays). We will briefly consider each condition.

\section{Tissue Handling}

Only by understanding key factors that can influence the phosphorylation state can we then gather data reflecting the true physiological phosphorylation status. The 2 most important factors appear to be time and fixative type. In the ideal setting, immediate snap freezing of cells would stop cellular processes such as phosphorylation. However, practicality and the suboptimal morphology of frozen sections make this impossible in a routine laboratory. Thus, fixed tissues must be used. Comparison of 5 commercially available fixatives showed that neutral buffered formalin fixation for durations typically used in clinical practice is satisfactory for quantitative assays [15]. Recently, a non-cross-linking (Streck; Omaha, Nebr., USA) tissue fixative was reported to significantly enhance the staining intensity of PPs compared with formalin [22]. Unfortunately, this fixative is costly and confirmatory studies are needed.

The time between tissue procurement and placement into the appropriate fixative may vary and affect PP levels in unpredictable ways due to the complexity of PP networks. Investigators have explored the issue of time to fixation. Using colon adenocarcinoma xenografts and IHC, the authors showed that pAKT levels were high in the samples fixed immediately in formalin but they were undetectable in tissues allowed to stand for $30 \mathrm{~min}$ at room temperature. Western blot (WB) analysis showed

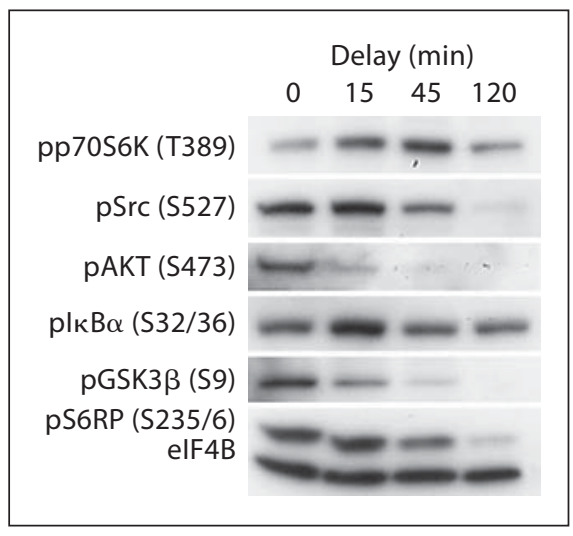

Fig. 2. Effect of freezing delay on PP levels in colon adenocarcinoma xenografts.

significant diminution of the signal by $15 \mathrm{~min}$ at room temperature [23]. We confirmed that already after $15 \mathrm{~min}$ of delay significant changes in PP levels can be detected (fig. 2). The fidelity of more than 50 signaling pathway PPs over time in tissue specimens procured in a community clinical practice has been reported [24]. Comparison across tissue specimens showed significant changes in PP levels (both increases and decreases) within $90 \mathrm{~min}$.

In an attempt to minimize these changes, investigators have suggested adding phosphatase and/or kinase inhibitors to a holding media or fixative [23-25]. While an attractive concept, the assumption is that such a cocktail completely inhibits all enzyme activity. Furthermore, because of complex networks and the interrelatedness of these types of signaling pathways, such additives cannot 'restore' the native PP patterns that may have been present in vivo after it has changed (prior to exposure to fixatives). Thus, the fidelity of initial PP levels may be lost if tissues are too large (causing problems of tissue penetration) or were not placed into appropriate fixatives within several minutes of removal from the patient.

In essence, the phrase 'time is the enemy' applies. Because of this, we contend that accurate PP detection must begin with ensuring a lack of significant delay in fixation. This can be achieved most easily in 2 settings. The first, which applied to the examples cited at the beginning of this article, is in small biopsies such as bone marrow trephine or endoscopic biopsies where the small biopsy sample is placed immediately into fixative at the bedside. The delay is measured in seconds and this is suitable for PP applications. The second situation is in the setting of fineneedle aspiration (FNA) cytology in which there would also be no delay between procurement of sample and 
placement into a fixative or preservative. Indeed the preparation of slides with individual cells or small cell clusters in liquid-based cytology (LBC) systems and the fact that tissue penetration is not an issue with FNA samples makes these samples an ideal substrate. Thus, an accurate 'snapshot' of PP status via the in situ detection of PPs is feasible. We have in fact embarked on studies to optimize specimen preparation to allow PP detection in LBC systems. Our preliminary data suggests this will be possible (fig. 3).

\section{Antibody Specificity}

In the clinical laboratory, we are 'at the mercy' of manufacturers. Regulations such as those governing analytespecific reagents (there are to our knowledge no FDAcleared PP antibodies for in vitro diagnostic use) are meant to ensure that such reagents are correctly manufactured, produced, and labeled. For most monoclonal antibodies in diagnostic use, we can confirm this since we have some idea based on positive and negative control tissues that antibodies are specific. Since most applications in routine IHC are lineage or cell-of-origin assignments, normal tissues with stable targets are often suitable. However, with functional phenotyping and the lability of epitopes, we believe an extra degree of diligence may be required of laboratory directors. For PPs, the use of cell lines manipulated to produce high and low levels (even complete absence) of intended targets or cell lines known to be positive and negative for particular PPs may be useful to confirm specificity. Such information is available for many targets $[15,26,27]$, but more published data is needed (see Generation of Robust Control Tissue Samples below). If the antibody is suitable for WB, simultaneous WB (cell lysates) and IHC or IF (cytospins or cell blocks) analyses can be performed. Alternatively, methods such as ELISA, intracellular flow cytometry [28], reverse-phase protein microarray [24], or mass spectrometry $[27,29]$ can prove the status of PP levels in the cells that can then be prepared for IHC or IF. Using these known positive and negative samples the staining antibody can then be shown to be specific for its target, followed by further confirmation in human tissues. Since little data exists on in situ PP patterns in tissues, one would need to use judgment, informed by knowledge of active biologic processes in various cell types, as to whether the antibody remains specific in tissues.

\section{Generation of Robust Control Tissue Samples}

As noted above, there is a great need for control tissue samples to allow laboratories to easily develop their as-
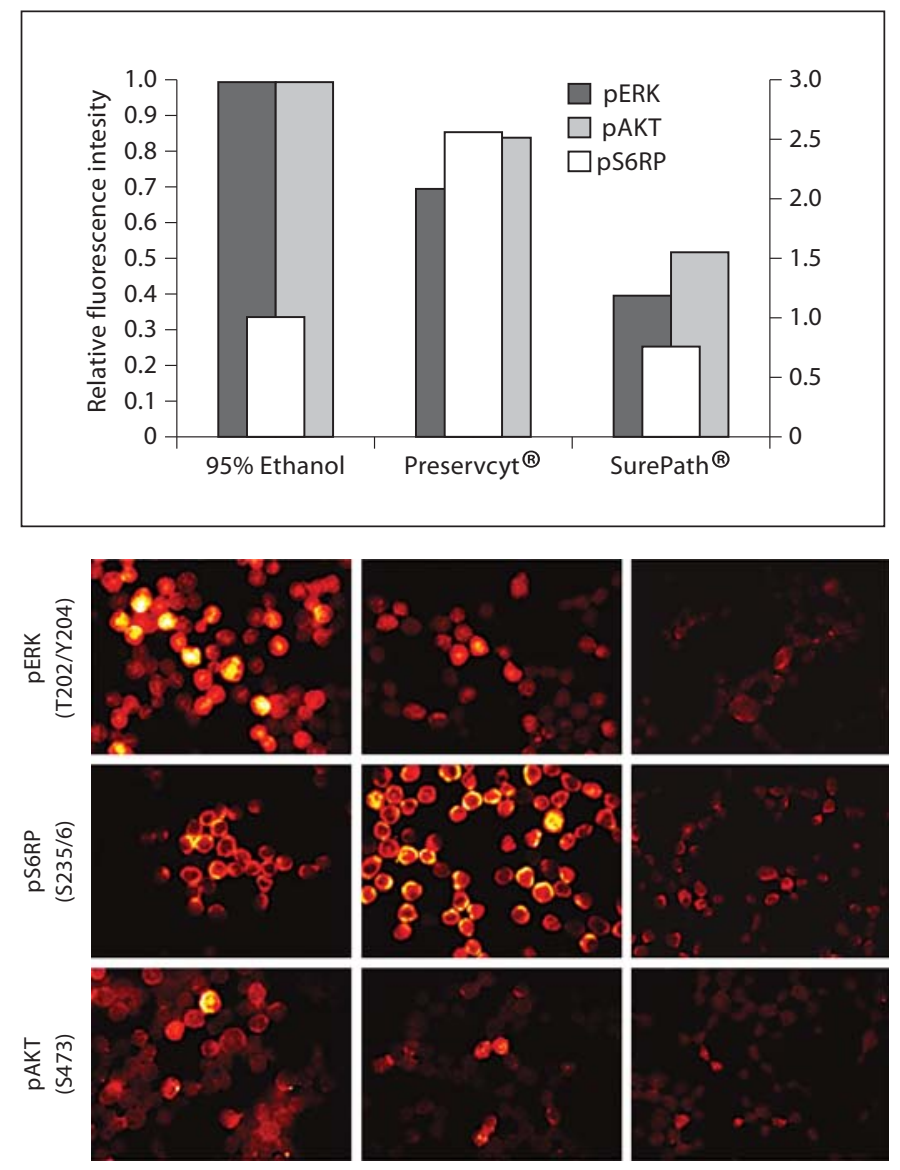

Fig. 3. Comparison of pERK, pS6RP, and pAKT levels in cells fixed in different preservatives used in LBC.

says. These could take several forms. We have chosen to develop a bank of cell lines that have been assayed for numerous PPs in a quantitative manner. Thus, for qualitative assays, one has available at least low/negative, midlevel, and high-level known control samples that assist in developing staining protocols. Cell lines have the advantage of relative stability, scalability, and uniformity. Once robust assays are developed, normal tissues (appropriately handled and fixed - see Tissue Handling above) can be screened and patterns established. This would likely be acceptable for qualitative assays. However, for quantitative assays, multilevel cell line standards and calibrators will be required. This type of quantitative assay is a new paradigm for clinical IHC laboratories. However, we believe this will be possible. For example, cell lines manipulated to express multiple levels of PPs can be assayed for absolute PP content/cell (on a population basis) and related to the quantitative IF level using image analysis (fig. 4). 

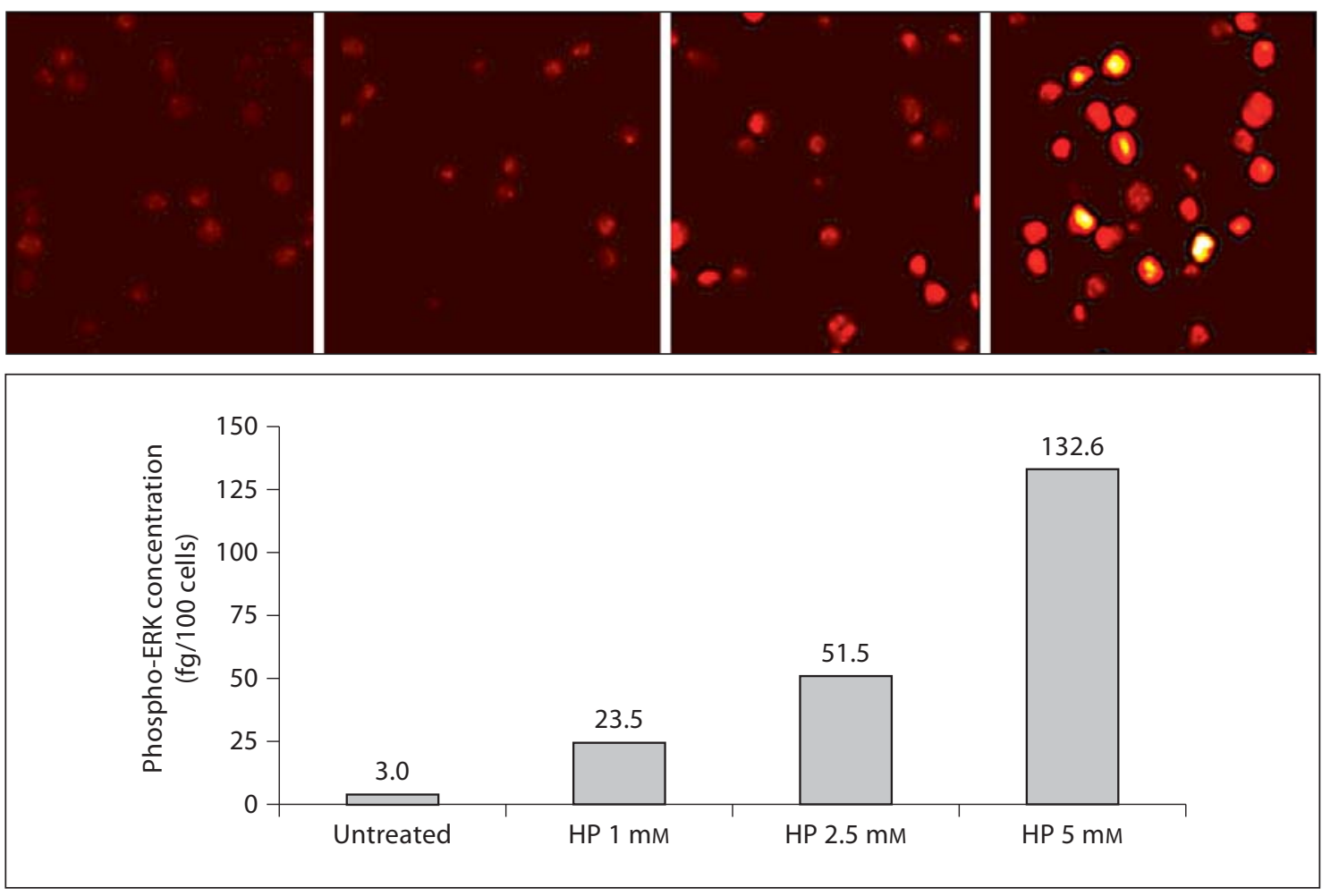

Fig. 4. Absolute quantification of pERK in a cell line treated with hydrogen peroxide. Quantum dot IF analysis is related to ELISA quantification.

\section{Selection of an Appropriate Imaging System for}

Quantitative Analysis

In order to maximize the amount of data from an assay, quantitative imaging systems that have a high sensitivity and facilitate multiplex assays should be used. A platform that has these qualities is multispectral imaging. In quantitative IF assays, multispectral imaging can minimize problems with the background (autofluorescence) and has a high sensitivity, thus increasing the potential dynamic range of an assay. Secondly, since this relies on the spectral characteristics rather than light at a particular wavelength band, multiplex assays are easier to achieve, particularly when fluorochromes are chosen, such as quantum dots, that have very narrow emission spectra and high quantum yields.

\section{Demonstration of Acceptable Performance}

\section{Characteristics}

Performance characteristics required for a functional phenotyping assay in tissues or cell preparations will depend, of course, on the intended use. For typical diagnostic applications of the type used currently in diagnostic immunohistochemical assays, typical descriptive data and Bayesian analyses are useful so that one knows the diagnostic utility and patterns in various tissues and tumor types [30]. However, for quantitative assays that may have therapeutic implications, one will need to demonstrate linearity in the biologically relevant range, limit of detection, and technical variability (CV of the assay). The existence of quantitative standards and calibrators will make these determinations possible. Finally, a successful assay will also depend on reliable sources of key reagents including primary antibodies and detection reagents.

\section{Conclusions}

Despite increasing knowledge of PPs, few PPs that fulfill the criteria to be used as biomarkers have been identified so far. In the era of targeted therapy, such markers are urgently needed. Unfortunately, most of the potential PP studies have not been reproduced. The variability between studies probably has multiple causes including the 
use of unvalidated techniques as well as differences in sample procurement, processing, and scoring/quantification. Furthermore, subjectively selected cutoffs may differ from one study to another and potentially lead to discrepant results. Thus there is a need for the rigorous standardization of laboratory procedures for tissue preservation and PP analysis. It represents a new paradigm for pathologists. Success in addressing the issues touched upon in this article will open a new era in functional phenotyping in which pathologists should play a major role.

\section{References}

$\checkmark 1$ Burnett G, Kennedy EP: The enzymatic phosphorylation of proteins. J Biol Chem 1954;211:969-980.

2 Mandell JW: Phosphorylation state-specific antibodies: applications in investigative and diagnostic pathology. Am J Pathol 2003;163: 1687-1698.

3 Yamaoka K, Saharinen P, Pesu M, Holt VE 3rd, Silvennoinen O, O'Shea JJ: The Janus kinases (Jaks). Genome Biol 2004;5:253.

4 Reiter A, Walz C, Watmore A, et al: The $\mathrm{t}(8$; 9)(p22;p24) is a recurrent abnormality in chronic and acute leukemia that fuses PCM1 to JAK2. Cancer Res 2005;65:2662-2667.

$\checkmark 5$ James C, Ugo V, Le Couedic JP, et al: A unique clonal JAK2 mutation leading to constitutive signalling causes polycythaemia vera. Nature 2005;434:1144-1148.

6 Levine RL, Wadleigh M, Cools J, et al: Activating mutation in the tyrosine kinase JAK2 in polycythemia vera, essential thrombocythemia, and myeloid metaplasia with myelofibrosis. Cancer Cell 2005;7:387-397.

-7 Aboudola S, Murugesan G, Szpurka H, et al Bone marrow phospho-STAT5 expression in non-CML chronic myeloproliferative disorders correlates with JAK2 V617F mutation and provides evidence of in vivo JAK2 activation. Am J Surg Pathol 2007;31:233-239.

8 Gibson SE, Schade AE, Szpurka H, Bak B, Maciejewski JP, Hsi ED: Phospho-STAT5 expression pattern with the MPL W515L mutation is similar to that seen in chronic myeloproliferative disorders with JAK2 V617F. Hum Pathol 2008;39:1111-1114.

-9 Zhang S, Fukuda S, Lee Y, et al: Essential role of signal transducer and activator of transcription (Stat)5a but not Stat5b for Flt3-dependent signaling. J Exp Med 2000;192:719728.

10 Abu-Duhier FM, Goodeve AC, Wilson GA, Care RS, Peake IR, Reilly JT: Genomic structure of human FLT3: implications for mutational analysis. Br J Haematol 2001;113: 1076-1077.

11 Thiede C, Steudel C, Mohr B, et al: Analysis of FLT3-activating mutations in 979 patients with acute myelogenous leukemia: association with FAB subtypes and identification of subgroups with poor prognosis. Blood 2002; 99:4326-4335.
12 Yokota S, Kiyoi H, Nakao M, et al: Internal tandem duplication of the FLT3 gene is preferentially seen in acute myeloid leukemia and myelodysplastic syndrome among various hematological malignancies: a study on a large series of patients and cell lines. Leukemia 1997;11:1605-1609.

13 Gilliland DG, Griffin JD: Role of FLT3 in leukemia. Curr Opin Hematol 2002;9:274281.

14 Bunting KD, Xie XY, Warshawsky I, Hsi ED: Cytoplasmic localization of phosphorylated STAT5 in human acute myeloid leukemia is inversely correlated with Flt3-ITD. Blood 2007;110:2775-2776.

15 Bodo J, Durkin L, Hsi ED: Quantitative in situ detection of phosphoproteins in fixed tissues using quantum dot technology. J Histochem Cytochem 2009;57:701-708.

16 Zuluaga TT, Hsieh FH, Bodo J, Dong HY, Hsi ED: Detection of phospho-STAT5 in mast cells: a reliable phenotypic marker of systemic mast cell disease that reflects constitutive tyrosine kinase activation. $\mathrm{Br} \mathrm{J}$ Haematol 2007;139:31-40.

17 Luo FR, Barrett YC, Yang Z, et al: Identification and validation of phospho-SRC, a novel and potential pharmacodynamic biomarker for dasatinib (SPRYCEL), a multi-targeted kinase inhibitor. Cancer Chemother Pharmacol 2008;62:1065-1074.

18 Chen Z, Lee FY, Bhalla KN, Wu J: Potent inhibition of platelet-derived growth factorinduced responses in vascular smooth muscle cells by BMS-354825 (dasatinib). Mol Pharmacol 2006;69:1527-1533.

19 Schittenhelm MM, Shiraga S, Schroeder A, et al: Dasatinib (BMS-354825), a dual SRC/ ABL kinase inhibitor, inhibits the kinase activity of wild-type, juxtamembrane, and activation loop mutant KIT isoforms associated with human malignancies. Cancer Res 2006;66:473-481.

20 Talpaz M, Shah NP, Kantarjian H, et al: Dasatinib in imatinib-resistant Philadelphia chromosome-positive leukemias. N Engl J Med 2006;354:2531-2541.
1 Tokarski JS, Newitt JA, Chang CY, et al: The structure of dasatinib (BMS-354825) bound to activated $\mathrm{ABL}$ kinase domain elucidates its inhibitory activity against imatinib-resistant ABL mutants. Cancer Res 2006;66: 5790-5797.

22 Burns JA, Li Y, Cheney CA, et al: Choice of fixative is crucial to successful immunohistochemical detection of phosphoproteins in paraffin-embedded tumor tissues. J Histochem Cytochem 2009;57:257-264.

23 Baker AF, Dragovich T, Ihle NT, Williams R, Fenoglio-Preiser C, Powis G: Stability of phosphoprotein as a biological marker of tumor signaling. Clin Cancer Res 2005;11: 4338-4340.

24 Espina V, Edmiston KH, Heiby M, et al: A portrait of tissue phosphoprotein stability in the clinical tissue procurement process. Mol Cell Proteomics 2008;7:1998-2018.

25 Patstone G, Maher PA: Phosphotyrosinecontaining proteins are concentrated in differentiating cells during chicken embryonic development. Growth Factors 1993;9:243252.

26 Fantin VR, Loboda A, Paweletz CP, et al: Constitutive activation of signal transducers and activators of transcription predicts vorinostat resistance in cutaneous T-cell lymphoma. Cancer Res 2008;68:3785-3794.

27 Rikova K, Guo A, Zeng Q, et al: Global survey of phosphotyrosine signaling identifies oncogenic kinases in lung cancer. Cell 2007; 131:1190-1203.

28 Krutzik PO, Irish JM, Nolan GP, Perez OD: Analysis of protein phosphorylation and cellular signaling events by flow cytometry: techniques and clinical applications. Clin Immunol 2004;110:206-221.

29 Zheng H, Hu P, Quinn DF, Wang YK: Phosphotyrosine proteomic study of interferon alpha signaling pathway using a combination of immunoprecipitation and immobilized metal affinity chromatography. Mol Cell Proteomics 2005;4:721-730.

30 Hsi ED: A practical approach for evaluating new antibodies in the clinical immunohistochemistry laboratory. Arch Pathol Lab Med 2001;125:289-294. 